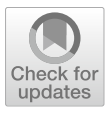

Cite as

Nano-Micro Lett.

(2021) $13: 153$

Received: 9 April 2021

Accepted: 15 June 2021

Published online: 8 July 2021

(c) The Author(s) 2021

\section{Interface Engineered Microcellular Magnetic Conductive Polyurethane Nanocomposite Foams for Electromagnetic Interference Shielding}

\author{
Guolong Sang ${ }^{1}$, Pei Xu ${ }^{1}$, Tong Yan ${ }^{1}$, Vignesh Murugadoss ${ }^{2,3}$, Nithesh Naik ${ }^{4}$, \\ Yunsheng Ding ${ }^{1}$, Zhanhu Guo ${ }^{3}$
}

\title{
HIGHLIGHTS
}

- Carbon nanotubes/polymerizable ionic liquid copolymer (CNTs/PIL) provides nucleation sites and inhibits the combination of microcellular structures.

- The increase in evaporate time improves the conductive network of composite foams.

- Electromagnetic interference shielding effectiveness (EMI SE) and specific EMI SE of the composite foam displays $69.9 \mathrm{~dB}$ and $211.5 \mathrm{~dB} /\left(\mathrm{g} \mathrm{cm}^{-3}\right)$.

- Polarization, conduction and magnetic loss attenuate microwave energy.

\begin{abstract}
Lightweight microcellular polyurethane (TPU)/ carbon nanotubes (CNTs)/ nickel-coated CNTs (Ni@CNTs)/ polymerizable ionic liquid copolymer (PIL) composite foams are prepared by non-solvent induced phase separation (NIPS). CNTs and Ni@CNTs modified by PIL provide more heterogeneous nucleation sites and inhibit the aggregation and combination of microcellular structure. Compared with TPU/CNTs, the TPU/CNTs/PIL and TPU/CNTs/Ni@CNTs/PIL composite foams with smaller microcellular structures have a high electromagnetic interference shielding effectiveness (EMI SE). The evaporate time
\end{abstract}

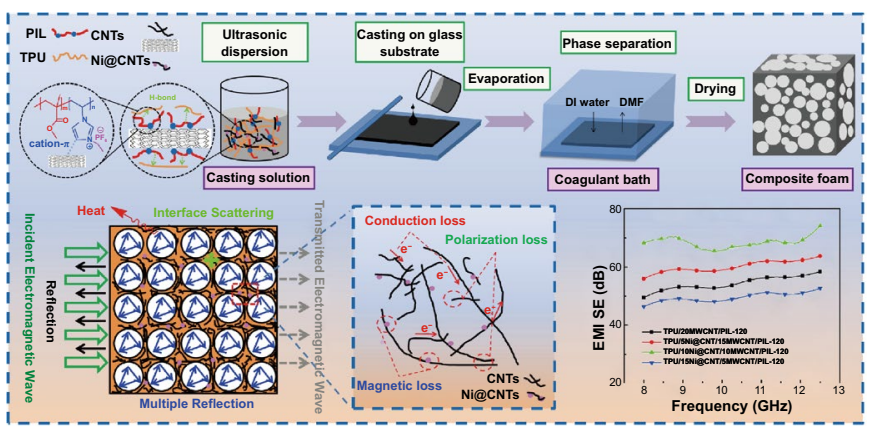
regulates the microcellular structure, improves the conductive network of composite foams and reduces the microcellular size, which strengthens the multiple reflections of electromagnetic wave. The TPU/10CNTs/10Ni@CNTs/PIL foam exhibits slightly higher SE values (69.9 dB) compared with TPU/20CNTs/PIL foam (53.3 dB). The highest specific EMI SE of TPU/20CNTs/PIL and TPU/10CNTs/10Ni@ CNTs/PIL reaches up to 187.2 and $211.5 \mathrm{~dB} /\left(\mathrm{g} \mathrm{cm}^{-3}\right)$, respectively. The polarization losses caused by interfacial polarization between TPU substrates and conductive fillers, conduction loss caused by conductive network of fillers and magnetic loss caused by Ni@CNT synergistically attenuate the microwave energy.

KEYWORDS Polyurethane; Nanocomposites; Microcellular; Electromagnetic interference shielding

$\checkmark$ Pei Xu, chxuper@hfut.edu.cn; Zhanhu Guo, zguo10@utk.edu

1 Department of Polymer Science and Engineering, School of Chemistry and Chemical Engineering, and Anhui Key Laboratory of Advanced Functional Materials and Devices, Hefei University of Technology, Hefei 230009, People's Republic of China

2 Advanced Materials Division, Engineered Multifunctional Composites (EMC) Nanotech. LLC, Knoxville, TN 37934, USA

3 Integrated Composites Laboratory (ICL), Department of Chemical and Biomolecular Engineering, University of Tennessee, Knoxville, TN 37996, USA

4 Department of Mechanical and Manufacturing Engineering, Manipal Institute of Technology, Manipal Academy of Higher Education, Manipal, Karnataka 576104, India 


\section{Introduction}

With extensive deployment and development of electronic equipment and communication devices, serious electromagnetic interference (EMI) interrupts the functions of equipment and affects the human organs [1-6]. Recently, more efforts have been made to seek for novel lightweight and high-performance EMI shielding materials [7-12]. Conductive polymer composites (CPCs) containing conductive nanofillers have excellent properties such as lightweight, excellent processability and resistance to corrosion compared with traditional metal-based EMI shielding materials [13-16]. CPCs with porous structures can further lighten the matrix weight and enhance the electrical performance of the composites even at a lower loading of fillers [17-20]. In addition, the preparation methods of electrically CPCs for EMI shielding materials also exert a profound impact on the performance of EMI shielding effectiveness (SE) [21-23].

Phase inversion method, subcritical $\mathrm{CO}_{2}$ foaming, freezedrying, gas foaming, micro-molding and 3D printing have been widely used to prepare EMI shielding materials with porous structures [24-26]. Phase separation methods include non-solvent-induced phase separation (NIPS), thermally induced phase separation (TIPS) and vapor-induced phase separation (VIPS) [27, 28]. Since porous polymer materials can be prepared by phase separation methods, NIPS as the most commonly and widely employed technique can be used to fabricate EMI shielding materials with microcellular structures [29]. In NIPS method, a substrate is covered with a thin layer of polymer solution and then immersed in a non-solvent bath, which causes the phase separation between the polymer-rich and polymer-dilute phases due to thermodynamic instability of the solution [30, 31]. The matrix via gelation and/or solidification can be formed in a polymer-rich phase, whereas the pores can be formed in the polymer-dilute phase [32]. A facile NIPS method can be used to prepare lightweight microcellular polyetherimide (PEI)/graphene foams with a density of $0.3 \mathrm{~g} \mathrm{~cm}^{-3}$ and specific EMI SE of $44 \mathrm{~dB} /\left(\mathrm{g} \mathrm{cm}^{-3}\right)$ [33].

Multi-walled carbon nanotubes (CNTs), which exhibit an exceptional structure, outstanding mechanical and electrical properties, can provide excellent EMI SE for polymer composite foams [34-36]. However, CNTs are hard to be dispersed in the polymers because of their large specific surface area and strong internal interaction $[37,38]$. The modification methods of CNTs include physical noncovalent modification and chemical covalent approaches [39, 40]. For the chemical approaches, the modifier forms the covalent linkage with a skeleton of the CNT by means such as radical polymerization and click chemistry to enhance the dispersion and solubilization of the CNT. However, chemical treatment would damage the translational symmetry of CNT by changing $s p^{2}$ carbon atoms to $s p^{3}$ carbon atoms, and the properties of nanofillers, including both the electronic and transport properties, are influenced [41]. Noncovalent modification of CNTs such as surfactants, charge-transfer agents and ionic liquids (ILs) is suitable for the facile and mild approaches that may not disrupt the structure and electron characteristics of CNTs [42]. Specifically, CNTs are noncovalently functionalized with modifiers such as cetyltrimethyl ammonium bromide (CTAB), polyvinylpyrrolidone (PVP) and ionic liquid (IL) through $\pi$-cation stacking, $\pi$ - $\pi$ stacking, H-bonds and hydrophobic interactions. For example, Narayan et al. used PVP as a destacking cum stabilizer of graphene nanosheets. Nonionic surfactant (PVP) adsorbed on the surface of graphene forms a covering layer, thereby preventing contact agglomeration between graphenes. However, the large aspect ratio of CNTs has a certain degree of entanglement, so a stronger force is required to stabilize the dispersion [41, 43]. Poly(ionic liquid)s and polymerizable ionic liquid copolymer (PIL) containing anion-cation pairs have strong cation- $\pi$ physical interactions with CNTs, which make them promising candidates for noncovalent modification of CNTs [44-47]. PIL-modified CNTs can have a promoted dispersion and increase the electrical conductivity of polymer matrix composites. Compared with other modification technologies of dispersing CNTs in polymer, this method is simpler, more effective and environmentally friendly [48, 49]. For example, Bose et al. reported that imidazolium-based ILs were used to modify noncovalently CNTs through a $\pi$-cation stacking interaction. The improved dispersion of IL-CNTs enhanced the electrical conductivity and electromagnetic radiation of polyvinylidene difluoride (PVDF) blends significantly [50].

Thermoplastic polyurethane (TPU) has aroused more interests owing to its high tensile strength and flexibility, good abrasion resistance, excellent chemical and thermal resistance [51-55]. TPU is widely used in flexible displays, smart clothing, electronic textiles and durable elastomeric wheels because TPU mainly consists of hard segments and 
soft segments [56-58]. The addition of CNTs into TPU can improve the mechanical and electrical properties in the nonconducting matrix for EMI shielding [59, 60]. The lightweight microcellular CPCs are particularly desirable for practical EMI shielding applications. To obtain the lightweight EMI materials, foaming makes the processing procedure easier and improves the EMI shielding performance. For example, Zeng et al. assembled anisotropic porous TPU/ CNTs composite foams by the freeze-drying method, and the low-density porous composite with the loading of 76.2 wt $\%$ CNTs achieved a SE of about $50 \mathrm{~dB}$ [22]. Kim et al. used nickel-coated carbon fiber (NCCF)/CNTs hybrid fillers as conductive fillers to prepare electrically conductive PU composite foams [61]. The EMI SE of PUF/NCCF/CNTs composites was $24.7 \mathrm{~dB}$ because NCCF and CNTs synergistically contributed to the conductance and magnetic permeability loss. A lightweight microcellular PVDF composite foam containing $10 \mathrm{wt} \% \mathrm{Ni}$-chains showed an enhanced EMI SE $(26.8 \mathrm{~dB})$ due to the tailored microcellular structure and high-aspect-ratio magnetic-conductive Ni chains [62]. The metallic nickel nanoparticles and CNTs can synergistically enhance the electrical conductivity, magnetic permeability and dielectric loss of the fillers to the electromagnetic wave.

In this study, CNTs were noncovalently modified with imidazolium-based PIL by the cation- $\pi$ noncovalent bond interaction and composed with Ni-coated CNT (Ni@CNT) to form hybrid fillers. The TPU nanocomposite foams
(TPU/CNTs/Ni@CNT/PIL) with microcellular structures were produced by a non-solvent induced phase separation (NIPS), which was a simple and environmentally friendly method. Scheme 1 shows the formation of TPU nanocomposite foams via the NIPS. The effects of IL content, filler content and evaporation time on the microcellular structure and EMI shielding properties were explored in details. In addition, the mechanism of micropore structure regulation was emphasized, and the influences of micropore structure regulation on the electrical conductivity and the EMI SE were investigated. The EMI shielding mechanism of this system was disclosed considering the microstructures and the network-induced conduction, magnetic loss and polarization loss. This research provides a novel and efficient way to prepare lightweight and high-performance electromagnetic shielding materials.

\section{Experimental Section}

\subsection{Materials}

TPU particles were purchased from Bayer AG and were polyester-based thermoplastic TPU pellets (790) with a density of $1.21 \mathrm{~g} \mathrm{~cm}^{-3}$. CNTs and Ni@CNTs (10-20 nm in outer diameter, $10-30 \mu \mathrm{m}$ in length) were supplied by Nanjing XFNANO Materials Tech Co., Ltd., China.

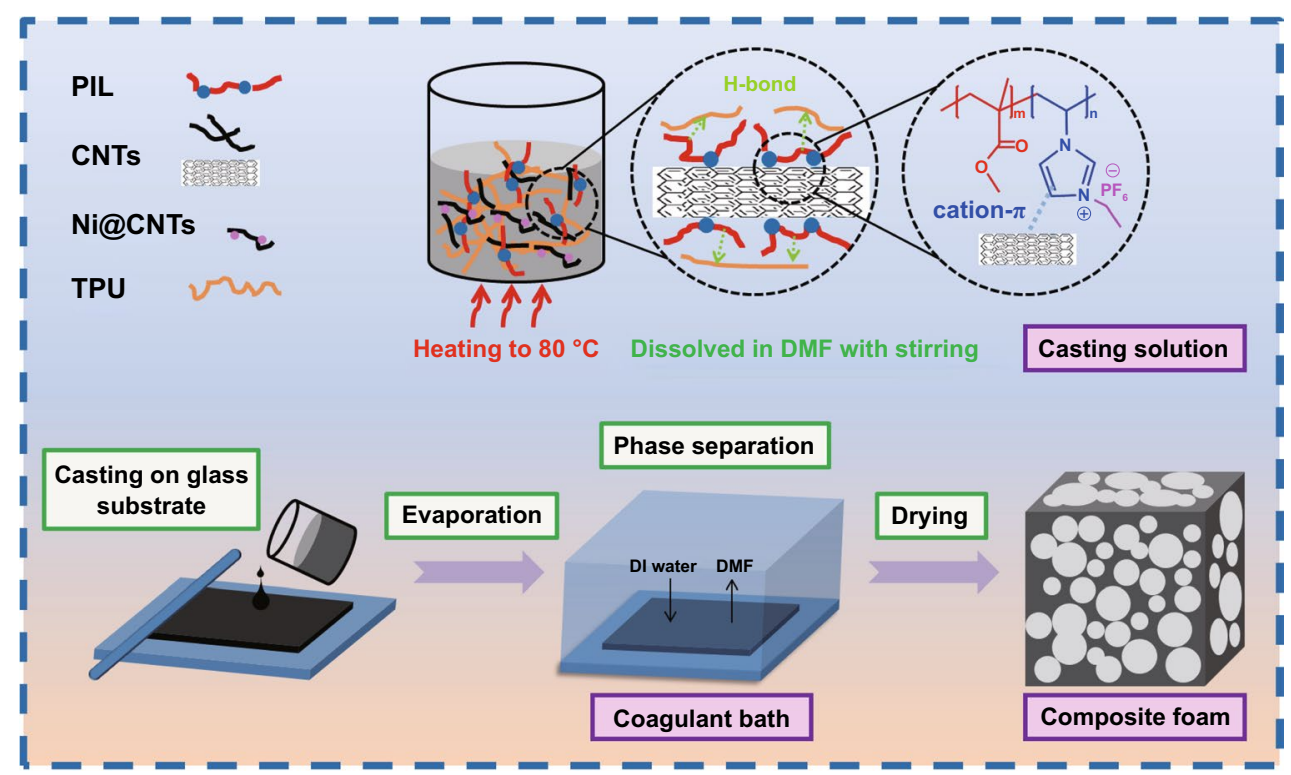

Scheme 1 Schematic for the formation of TPU/CNTs composite foams via the NIPS 
$N, N$ '-dimethylformamide (DMF) and other reagents were provided by Sinopharm Chemical Reagent (China). The synthesis and characterization of PIL are shown in Scheme S1, Figs. S1 and S2.

\subsection{Preparation of TPU Nanocomposite Foams via NIPS}

The fabrication of TPU nanocomposite foams is schematically illustrated in Scheme 1. Firstly, the TPU and PIL were dissolved in anhydrous DMF with magnetic stirring at $80{ }^{\circ} \mathrm{C}$ for $2 \mathrm{~h}$. Simultaneously, CNTs and/or Ni@CNTs were dispersed in the solution of PIL with the aid of sonication. The weight ratio of CNTs to PIL was 4 to 1 . The suspension of modified CNTs or pristine CNTs was added to the TPU solution, and the mixture was subjected to the ultrasonic treatment for $2 \mathrm{~h}$ to form a homogeneous slurry. The resulting slurry was degassed under vacuum and then casted on a pre-cleaned planar glass substrate. DMF was chosen as polymer solvent, whereas deionized (DI) water was used as a non-solvent. The casted film was allowed to evaporate for a certain time $(3,15,30,60,90$ and $120 \mathrm{~min})$ before immersed in the coagulation bath to induce phase inversion. The symbol " $\mathrm{x}$ " in the TPU/CNTs-x, TPU/ CNTs/PIL-x and TPU/CNTs/Ni@CNTs/PIL-x represents the evaporate time $(3,15,30,60,90$ and $120 \mathrm{~min})$. This step was crucial for forming a porous structure. Then, the casted film was immersed into the coagulation bath for $6 \mathrm{~h}$. In the casted film, porous structure was formed and solidified by phase separation process through the exchange of solvent (DMF) and non-solvent (water) in the coagulation bath. Finally, the TPU composite foams were obtained after washing with deionized water to remove the residual solvent and drying at $40{ }^{\circ} \mathrm{C}$ for $24 \mathrm{~h}$. Table 1 shows the formulation of TPU composite foams.

\subsection{Characterization}

The Raman spectroscopy was performed with a Raman spectrometer (LABRAM-HR), which is equipped with an excitation wavelength of $532 \mathrm{~nm}$. The XPS spectra of the samples were obtained via an X-ray photoelectron spectroscopy (ESCALAB250Xi, Thermo Fisher). The morphology of sample was observed by a scanning electron
Table 1 Formulation of TPU composite foams

\begin{tabular}{llllll}
\hline Sample & TPU & CNTs & Ni@CNTs & PIL & DMF \\
\hline TPU & 15 & - & - & - & 85 \\
TPU/10CNTs & 13.5 & 1.5 & - & - & 85 \\
TPU/15CNTs & 12.75 & 2.25 & - & - & 85 \\
TPU/20CNTs & 12 & 3 & - & - & 85 \\
TPU/25CNTs & 11.25 & 3.75 & - & - & 85 \\
TPU/10CNTs/PIL & 13.13 & 1.5 & - & 0.38 & 85 \\
TPU/15CNTs/PIL & 12.19 & 2.25 & - & 0.56 & 85 \\
TPU/20CNTs/PIL & 11.25 & 3 & - & 0.75 & 85 \\
TPU/25CNTs/PIL & 10.31 & 3.75 & - & 0.94 & 85 \\
TPU/15CNTs/5Ni@CNTs/ & 11.25 & 2.25 & 0.75 & 0.75 & 85 \\
PIL & & & & & \\
TPU/10CNTs/10Ni@CNTs/ & 11.25 & 1.5 & 1.5 & 0.75 & 85 \\
PIL & & & & & \\
TPU/5CNTs/10Ni@CNTs/ & 11.25 & 0.75 & 2.25 & 0.75 & 85 \\
PIL & & & & & \\
\hline
\end{tabular}

microscope (SEM, HITACHI, SU-8020). The density ( $\rho$ ) of microcellular foams can be calculated by the water displacement method. The electrical conductivity performance was determined using a multi-function digital electric meter by a homemade fixture (Victor Tech, Victor 86-e).

The EMI SE performance of TPU composite foams was measured using a vector network analyzer (N5247A, Agilent Technologies) in X band [63]. The corresponding dimension of samples was $22.86 \times 10.16 \times 2 \mathrm{~mm}^{3}$. The EMI shielding properties of the as-prepared materials were evaluated with $S$ parameters, which were used to calculate $\mathrm{SE}_{\mathrm{T}}$ (total shielding effectiveness), $\mathrm{SE}_{\mathrm{R}}$ (reflection loss) and $\mathrm{SE}_{\mathrm{A}}$ (absorption loss) [28].

\section{Results and Discussion}

\subsection{Characterization of CNTs and CNTs/PIL}

Figure 1a shows the Raman spectra of CNTs and CNTs/ PIL hybrids. Pristine CNTs generally show three characteristic peaks. The D-band $\left(1348 \mathrm{~cm}^{-1}\right)$ derives from amorphous carbon and lattice defects in the structure, the G-band $\left(1581 \mathrm{~cm}^{-1}\right)$ corresponds to the tangential vibrations of carbon atoms, and the 2D-band $\left(2697 \mathrm{~cm}^{-1}\right)$ is closely related to the electronic band structure of carbon nanomaterials [38]. The characteristic peaks of D, 
G and 2D-bond of CNTs/PIL appear at 1324, 1574 and $2650 \mathrm{~cm}^{-1}$, respectively. The blue shift of the characteristic peaks indicates that the electron density of CNTs is strengthened owing to the cation- $\pi$ interaction between imidazolium groups in PIL and $\pi$-electrons in CNTs. The increased intensity ratio of $I_{\mathrm{D}} / I_{\mathrm{G}}$ from 0.75 to 0.96 indicates that the cation- $\pi$ interaction affects the $\pi-\pi$ electronic conjugation and the increase in vibration energy [47].

The C $1 \mathrm{~s}$ spectra of CNTs and CNTs/PIL from the XPS analysis are shown in Fig. 1b. As shown in Fig. 1b, the C $1 \mathrm{~s}$ signal is reduced in the full scan XPS spectra of CNTs/ PIL sample, whereas there is an obvious presence of $\mathrm{F} 1 \mathrm{~s}, \mathrm{O}$ 1s and $\mathrm{N}$ ls elements because of the modification of CNTs by PIL. As calculated, the elemental concentrations ratio of $\mathrm{C}$ and $\mathrm{O}$ in the CNTs/PIL is 14.2, which is much lower than that of pristine CNTs (44.9) because of the cation- $\pi$ interactions [41]. The fitted curves of C $1 \mathrm{~s}$ spectra of CNTs and CNTs/PIL are shown in Fig. 1c, d, respectively. These C $1 \mathrm{~s}$ spectra can be deconvoluted into various sub-peaks with different binding energies: $s p^{2} \mathrm{C}=\mathrm{C}(284.8 \mathrm{eV}), s p^{3}$ $\mathrm{C}-\mathrm{C}(285.1 \mathrm{eV}), \mathrm{C}-\mathrm{N}$ at $285.7 \mathrm{eV}, \mathrm{C}-\mathrm{O}$ at $286.3 \mathrm{eV}, \mathrm{C}=\mathrm{O}$ at $287.2 \mathrm{eV}, \mathrm{O}-\mathrm{C}=\mathrm{O}$ at $288.3 \mathrm{eV}$ and $\pi-\pi^{*}$ at $290.9 \mathrm{eV}$. The XPS spectra data are displayed in Tables S1 and S2. Compared with CNTs, the C 1 s peaks of the CNTs/PIL hybrids appear to have two new sub-peaks at around 285.1 and $288.3 \mathrm{eV}$ because the $\pi-\pi$ electronic conjugation of pristine CNTs is affected by noncovalent modifications of the PIL [44]. All these results indicate that PIL has successfully modified CNTs by noncovalent cation- $\pi$ interaction, thereby improving their dispersion and compatibility in the polymer matrix.

\subsection{Morphology of TPU Composite Foams}

The typical SEM microstructures shown in Figs. 2 and S3 are used to explore the effect of CNTs modification by PIL on the morphology of TPU composite foams with 3 min evaporate time and different filler contents. The pore size distribution of TPU/10CNTs -3(a) and TPU/10CNT/ PIL-3(b) with 3 min evaporate time is shown in Fig. S4 and Table S1. The honeycomb microcellular structure of
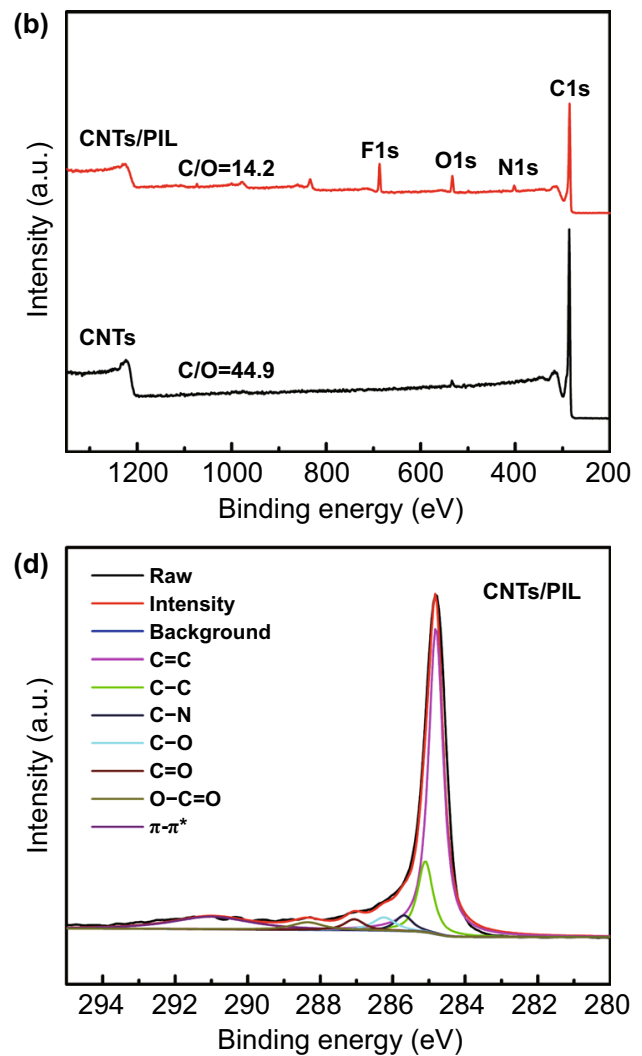

Fig. 1 Raman spectra a and XPS spectra $\mathbf{b}$ of CNTs and CNTs/PIL; C $1 \mathrm{~s}$ XPS spectra of CNTs $\mathbf{c}$ and $\mathbf{d}$ CNTs/PIL 
TPU/CNTs and TPU/CNTs/PIL foams was both welldefined when the filler loading was less than $20 \mathrm{wt} \%$ because the diffusion of water to casting solution of the composite can induce physical gelation and phase separation [64]. However, the honeycomb microcellular structure of TPU/20CNTs and TPU/25CNTs foams was destroyed when the filler loading was increased to 20 and $25 \mathrm{wt} \%$, because a higher content of CNTs led to the increased viscosity of the casting solution. After modifying CNTs via PIL, the cell size of TPU/CNTs/PIL composite foams is smaller and the microcellular structure is homogeneous with the same filler content compared with the TPU/ CNTs composite foams. The reason for this phenomenon is that PIL decreases the surface tension gradient at the surface of the non-solvent phase and thereby attenuates the Marangoni convection. This in turn decreases the mass-transfer and the size of solvent dilute phase [65]. In addition, the cation- $\pi$ interaction between PIL and CNTs improved the dispersion of CNTs in the casting solution and increased the heterogeneous nucleation sites. The uniform microcellular structure of TPU/25CNTs/PIL was slightly destroyed because of the higher viscosity of the suspension. The driving force induced by the solvent concentration is affected by the physical barrier of the high content filler, which inhibits the mass-transfer and forms irregular honeycomb microcellular structure $[32,66]$.

\subsection{Electrical and EMI Shielding Properties of TPU Composite Foams}

The EMI SE of TPU composite foams containing different filler contents with $2 \mathrm{~mm}$ thickness and $3 \mathrm{~min}$ evaporate time is shown in Fig. 3a, b. The EMI SE exhibits fluctuations with frequency because the microcellular structure in the entire composite material results in the non-uniformity
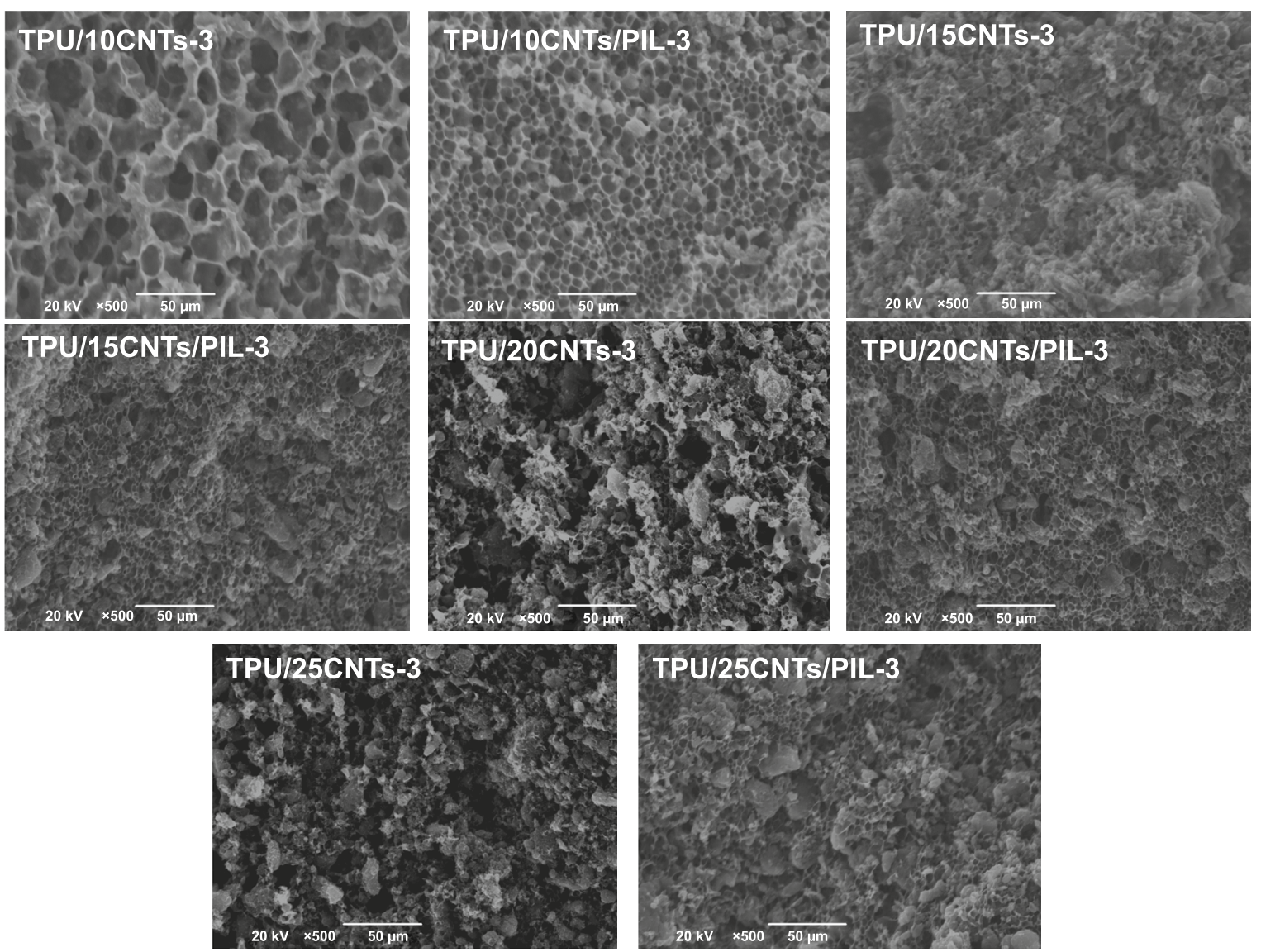

Fig. 2 Typical SEM images for fracture morphology of microcellular TPU/CNTs and TPU/CNTs/PIL composite foams with 3 min evaporate time 
of the discrete conductive network size [34]. The EMI SE value of TPU composite foams increases with increasing the filler content. As the filler content is increased to $20 \mathrm{wt} \%$, the EMI SE of TPU/20CNTs and TPU/20CNTs/PIL foams is improved to 25.5 and $27.2 \mathrm{~dB}$ at $9.0 \mathrm{GHz}$, respectively. By increasing the loading to $25 \mathrm{wt} \%$, the EMI SE value of TPU/25CNTs and TPU/25CNTs/PIL foams reaches 30.2 and $34.1 \mathrm{~dB}$, respectively. The TPU/CNTs/PIL composite foams have a higher EMI SE value because PIL coating on the surface of CNTs reduces the surface energy and enhances the interface between the substrates of TPU [67]. The modification of CNTs by PIL enhances the dispersion of CNTs and increases the viscosity of casting solution in the phase separation process. It provides more heterogeneous nucleation sites and inhibits the aggregation and combination of microcellular structure, which plays a role in regulating the microcellular morphology and reducing the size of microcellular [33, 34].

The $\mathrm{SE}_{\mathrm{T}}$ is mainly composed of $\mathrm{SE}_{\mathrm{A}}, \mathrm{SE}_{\mathrm{R}}$ and $\mathrm{SE}_{\mathrm{M}}$. $\mathrm{SE}_{\mathrm{M}}$ can be ignored when the $\mathrm{SE}_{\mathrm{T}}$ is greater than $15 \mathrm{~dB}$
[68]. To further illuminate the EMI shielding mechanism of the TPU composite foams, the measured scattering parameters are used to calculate the $\mathrm{SE}_{\mathrm{A}}$ and $\mathrm{SE}_{\mathrm{R}}$ at 9.0 GHz as shown in Fig. 3c, d. Clearly, the $\mathrm{SE}_{\mathrm{A}}$ and $\mathrm{SE}_{\mathrm{R}}$ of composite foams increase with increasing the filler content. In addition, the $\mathrm{SE}_{\mathrm{R}}$ occupies a small proportion of the $\mathrm{SE}_{\mathrm{T}}$ and the $\mathrm{SE}_{\mathrm{A}}$ is the main EMI shielding mechanism in the X-band for TPU composite foams. The multiple reflections of electromagnetic waves caused by the microcellular structure and conductive network in the foams converted the microwave power into heat dissipation [52]. The absorption efficiency of TPU/CNTs/PIL is slightly higher than that of TPU/CNTs. The absorption originates from the electrical conductivity of CNTs, and polarization loss between TPU substrates and conductive fillers. It can be considered that the smaller microcellular structure and more uniformly dispersed CNTs enhance the multiple reflection and energy conversion through conductivity and polarization loss [69].
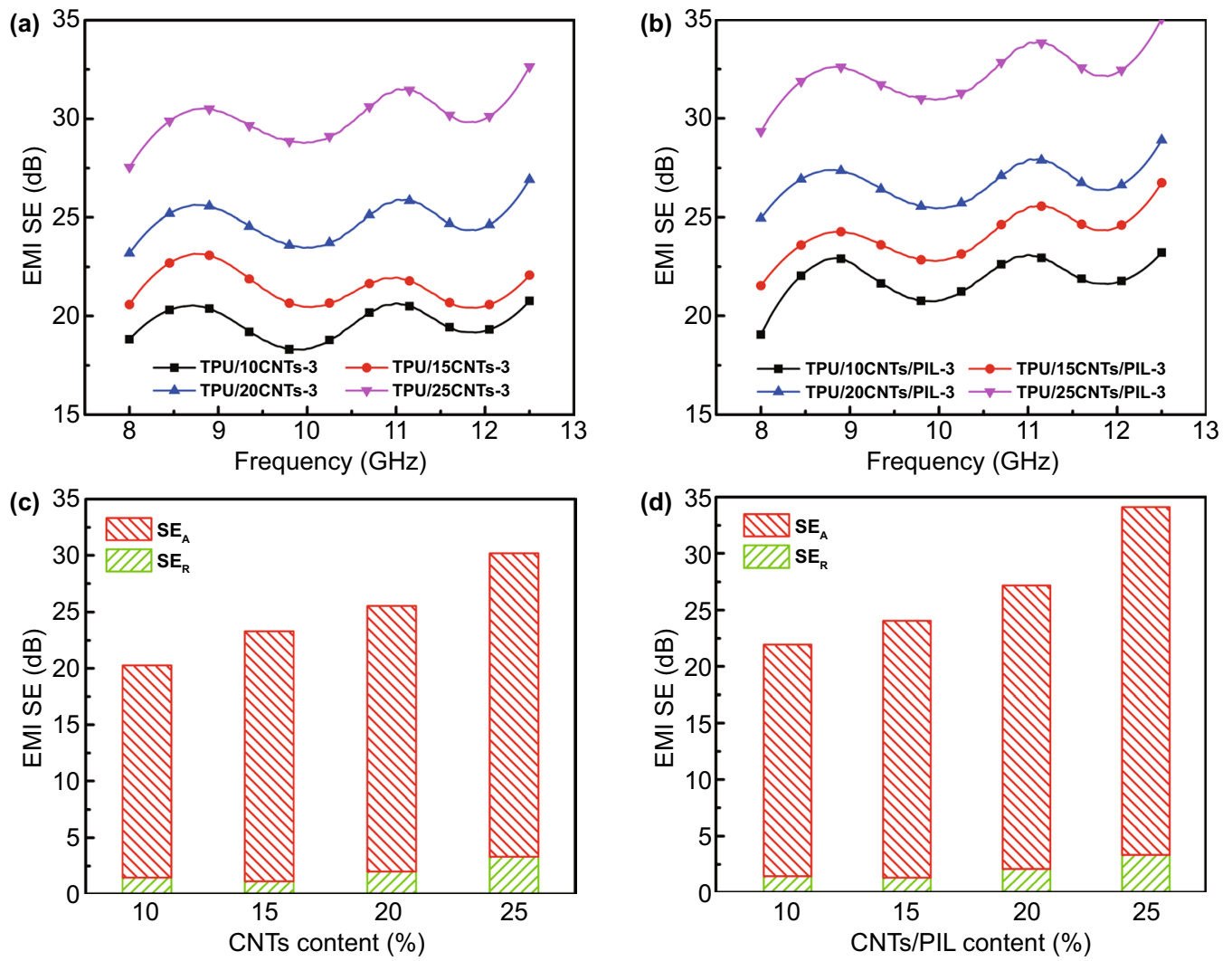

Fig. 3 EMI SE of TPU/CNTs a and TPU/CNTs/PIL b composite foams with 3 min evaporate time and various concentrations; $\mathrm{SE}_{\mathrm{A}}$ and $\mathrm{SE}_{\mathrm{R}}$ of TPU/CNTs $\mathbf{c}$ and TPU/CNTs/PIL $\mathbf{d}$ composite foams with $3 \mathrm{~min}$ evaporate time and various concentrations at $9.0 \mathrm{GHz}$ 


\subsection{Effect of Evaporate Time on Morphology of TPU Composite Foams}

Evaporate time refers to the time interval between the casting solution pouring into the mold and immersing it in the coagulation bath. The effect of evaporate time on the kinetics and thermodynamic equilibrium of phase separation process was revealed by SEM. As shown in Figs. 4 and S5, the morphology of composite foams gradually becomes dense with the increase in the evaporation time. In contrast, the morphology of TPU/20CNTs is rough and has many voids because of the increased viscosity and the introduced physical barrier of CNTs. The microcellular structure of PU/CNT composite is almost completely destroyed when the evaporation time exceeds $30 \mathrm{~min}$. But the microcellular structure of TPU/20CNTs/PIL composites is not destroyed and becomes more uniform compared with that of TPU/20CNTs [53].

The morphological differences can be attributed to the effects of PIL and evaporation time during the NIPS process. Firstly, PIL-modified CNTs improved the dispersion of CNTs, acted as nucleation agents and provided numerous nucleation sites for the NIPS foaming process. Subsequently, PIL as a surfactant can be dissolved in the
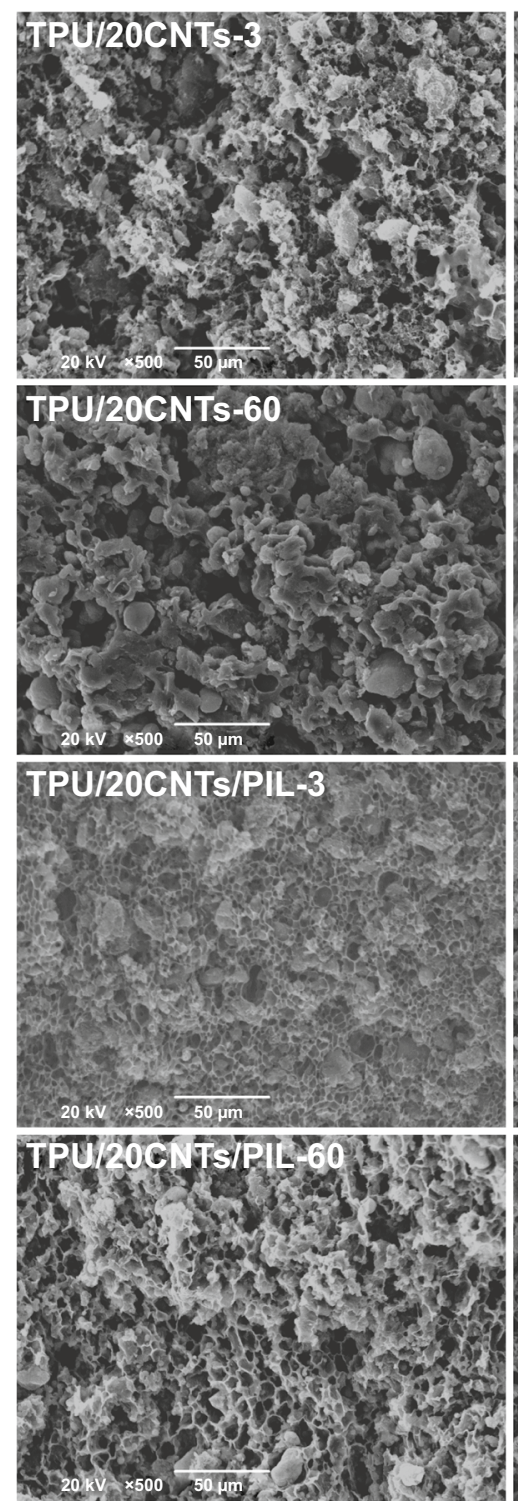
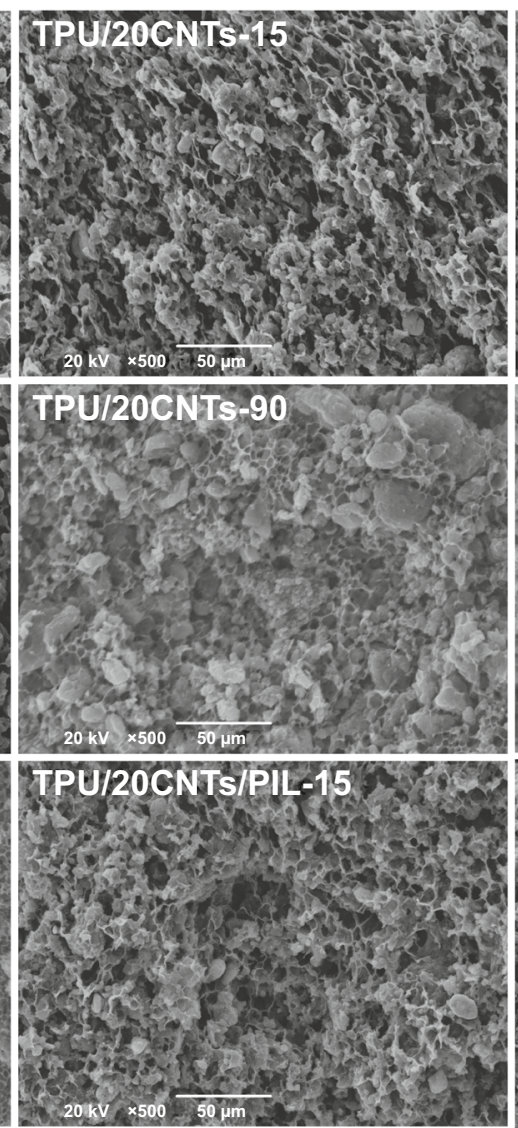

TPU/20बNTSIPIE-90

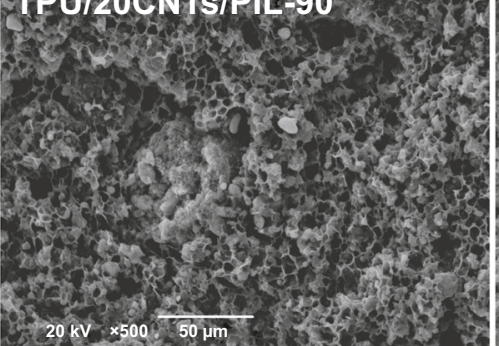

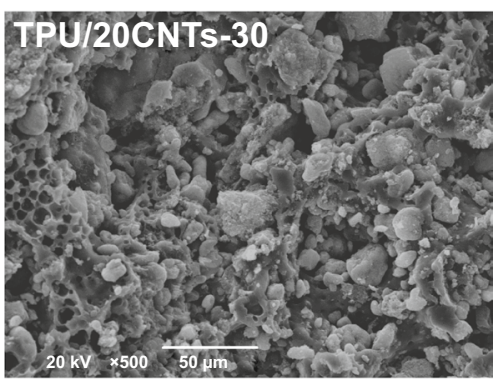
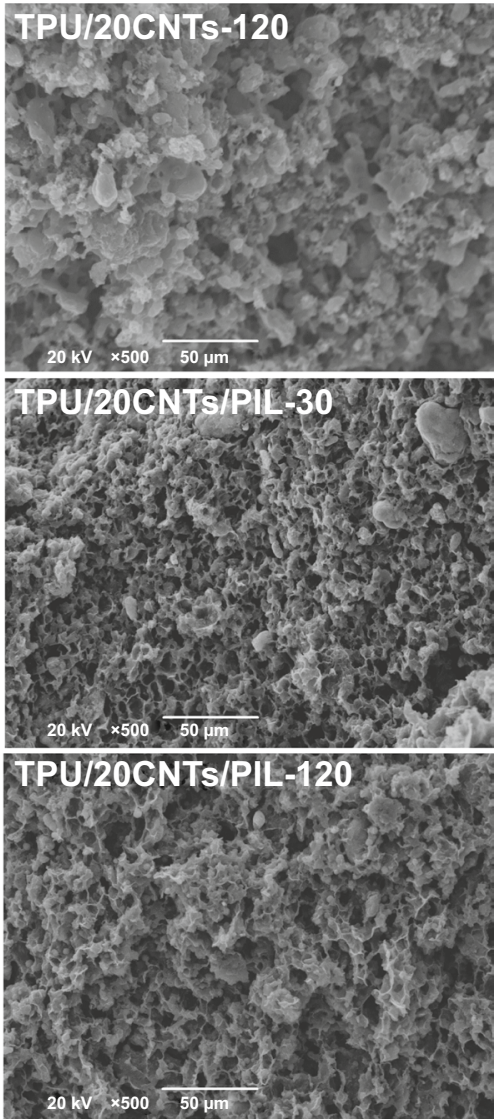

Fig. 4 SEM images of TPU/20CNTs and TPU/20CNTs/PIL composite foams with different evaporate time (3, 15, 30, 60, 90 and 120 min) 
solvent, decreases the surface tension of casting solution, and then the agglomeration of DI-rich droplets at the liquid/air interface is hindered [30]. Finally with increasing the evaporate time, the viscosity of casting solution is increased and the formed physical barrier leads to the cell agglomeration.

\subsection{Effect of Evaporate Time on Electrical and EMI Shielding Properties}

Figure 5a compares the electrical conductivity of TPU/ CNTs and TPU/CNTs/PIL composite foams with different evaporate time. It can be seen that the electrical conductivity of TPU/20CNTs/PIL-3 $\left(18.9 \mathrm{~S} \mathrm{~m}^{-1}\right)$ is 2 times that of TPU/20CNTs-3 $\left(9.4 \mathrm{~S} \mathrm{~m}^{-1}\right)$. The electrical conductivity of TPU/20CNTs/PIL-15 $\left(31.2 \mathrm{~S} \mathrm{~m}^{-1}\right)$ is 1.5 times that of TPU/20CNTs-15 (20.1 S m $\left.\mathrm{S}^{-1}\right)$. When the evaporation time exceeds $30 \mathrm{~min}$, the electrical conductivity of the TPU/20CNTs and TPU/20CNTs/PIL is increased slowly and is of the same order of magnitude. It can be found that the electrical conductivity of TPU/20CNTs/PIL is higher than that of TPU/20CNTs. It is indicated that the conductive network in the composites is gradually improved due to the improved dispersion and distribution of the conductive fillers with increasing the evaporation time. These proved that PIL is helpful to improve the dispersion of CNTs through the noncovalent modification. With the extension of evaporation time, the solid-liquid phase separation induced the conductive filler to be distributed in the rich polymer phase, which further improved the conductive network of composite foams and finally reached saturation. In summary, the noncovalent modification of CNTs by PIL and the distribution of conductive fillers regulated by solid-liquid phase separation have significantly improved the electrical conductivity of the foams [70].

The densities of the TPU/20CNTs and TPU/20CNTs/PIL composite foams with different evaporate time are shown in Fig. 5b. It can be seen that the density of both composite foams increases with the extension of evaporate time. It
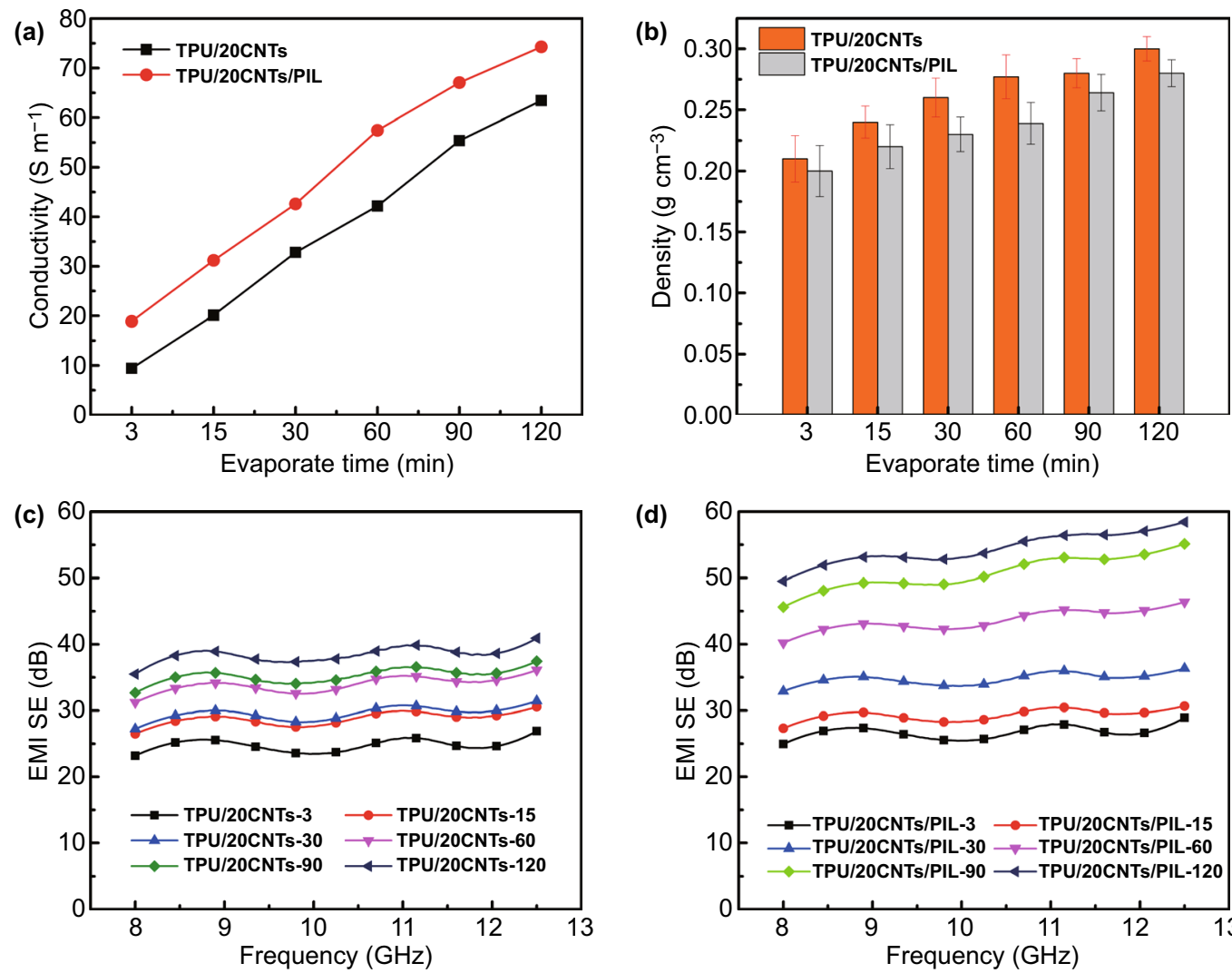

(d)

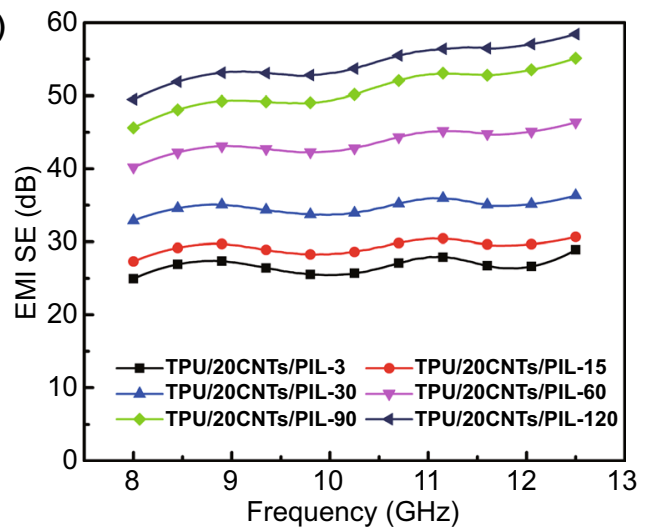

Fig. 5 Conductivity $\mathbf{a}$ and density $\mathbf{b}$ of TPU composite foams with different evaporate time; $\mathbf{c}$ and $\mathbf{d}$ EMI SE of TPU composite foams 
should be noted that the modification of CNTs by PIL significantly decreases the density of composite foams. The presence of PIL optimizes the dispersion of CNTs and increases the viscosity of the casting solution. This in turn results in uniform cells and reduces mass transfer and density of the composite foam [58].

Figure $5 \mathrm{c}, \mathrm{d}$ shows the EMI SE of the TPU/20CNTs and TPU/20CNTs/PIL foams. It is seen that the EMI SE of TPU/CNTs and TPU/CNTs/PIL is gradually increased as the evaporation time is increased from 3 to $120 \mathrm{~min}$. The conductive network is improved with the increase in micro-phase separation, which provides more pathways for the transition and transmission of free electrons. The results indicate that the solid-liquid phase separation improves the conductive network of composite foams and reduces the microcellular size, which strengthens the multiple reflection of electromagnetic wave [71]. In addition, compared with the TPU/CNTs composite foams, the addition of PIL in TPU/CNTs/PIL had given a higher EMI SE value under the same condition. The maximum EMI SE of TPU/20CNTs/ PIL reaches $53.3 \mathrm{~dB}$ because the PIL improves the dispersion of CNTs and provides more transmission of free electrons and stronger interfacial polarization. Uniform microcellular structure and perfect conductive network had a large number of electrons or holes acting as carriers of mobile charges [28]. Under the action of external electromagnetic waves, these carriers are transmitted along the conductive network and then convert the EM energy into the thermal energy.

In order to further illuminate the influences of filler content and evaporate time on the EMI SE of composite foams, various filler concentrations were investigated when the evaporate time was fixed at $120 \mathrm{~min}$. The results are shown in Fig. 6. The EMI SE values of TPU/CNTs and TPU/ CNTs/PIL composite foams are still positively correlated with the filler concentration. The EMI SE of TPU/25CNTs and TPU/25CNTs/PIL composite foams is 40.6 and $54.6 \mathrm{~dB}$, respectively, when the filler content is $25 \mathrm{wt} \%$. The EMI $\mathrm{SE}$ of composite foams is increased significantly with the increase in filler concentration. However, the EMI SE of TPU/CNTs/PIL composite foam is stabilized at $60 \mathrm{~dB}$ without any significant increase when the filler concentration is beyond $20 \mathrm{wt} \%$. This is because the conductive network has been substantially improved when the filler concentrations are $20 \mathrm{wt} \%$. The further increase in filler concentration has a weak strengthening effect on the conductive network and may even destroy the microcellular structure of the composite foams [72]. These results are consistent with that from SEM images (Fig. 2). Therefore, the EMI shielding performance of composite foams mainly originates from the formed interconnected CNTs networks and the porous structure that attenuate the incident electromagnetic waves via multiple reflections [73].

The EMI SE of TPU/CNTs/Ni@CNTs/PIL foams with $2.0 \mathrm{~mm}$ thickness are shown in Fig. 7a. The EMI SE of TPU/CNTs/Ni@CNTs/PIL foams is firstly increased and then reduced with increasing the Ni@CNTs content. The electrical conductivity of TPU/15CNTs/5Ni@CNTs/PIL, TPU/10CNTs/10Ni@CNT/PIL and TPU/5CNTs/15Ni@ CNTs/PIL foams with $3 \mathrm{~min}$ are 15.3, 12.2 and $8.7 \mathrm{~S} \mathrm{~m}^{-1}$, respectively. The electrical conductivity of TPU/20CNTs/ PIL with $3 \mathrm{~min}$ is $18.9 \mathrm{~S} \mathrm{~m}^{-1}$. The electrical conductivity of
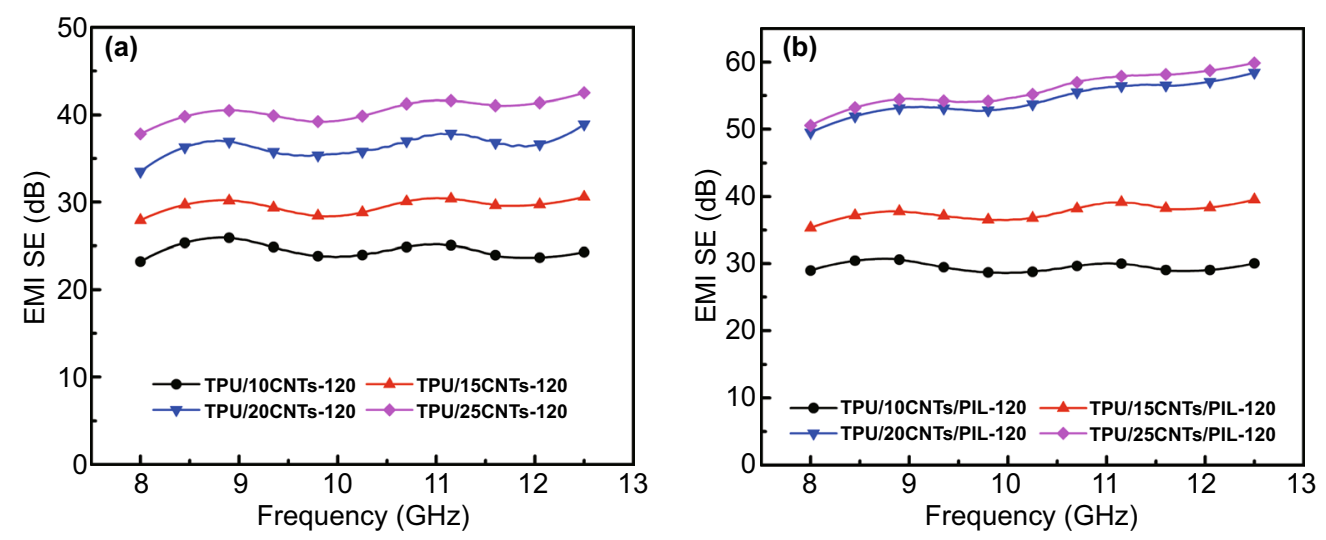

Fig. 6 EMI SE of TPU/CNTs and TPU/CNTs/PIL composite foams with various filler concentrations and an evaporate time of 120 min 
TPU/15CNTs/5Ni@CNTs/PIL,TPU/10CNTs/10Ni@CNT/ PIL and TPU/5CNTs/15Ni@CNTs/PIL foams with $120 \mathrm{~min}$ is $65.3,56.8$ and $43.7 \mathrm{~S} \mathrm{~m}^{-1}$, respectively. The electrical conductivity of TPU/20CNTs/PIL with $120 \mathrm{~min}$ is $74.2 \mathrm{~S}$ $\mathrm{m}^{-1}$. The electrical conductivity of PU/CNTs/Ni@CNTs/ PIL foams with the same filler content is slightly lower than that of PU/20CNTs/PIL because the electrical conductivity of CNTs is slightly lower than that of Ni@CNTs. The EMI SE of TPU/10CNTs/10Ni@CNTs/PIL foam is $69.9 \mathrm{~dB}$ at 10 wt\% Ni@CNTs and 10 wt\% CNTs content (Fig. S6 shows the Raman spectra and XPS spectra of CNTs/Ni@CNTs/ PIL, Tables S1 and S2 show the date of XPS spectra). The EMI SE of TPU/20CNTs/PIL foam is slightly lower than that of TPU/10CNTs/10Ni@CNTs/PIL foam.

Figure $7 \mathrm{~b}$ shows the $\mathrm{SE}_{\mathrm{T}}, \mathrm{SE}_{\mathrm{R}}$ and $\mathrm{SE}_{\mathrm{A}}$ at $9 \mathrm{GHz}$ of all foams. It is indicated that the absorption mainly contributes to the EMI SE for all foams. The multi-pore microstructure of the foams effectively relieves the impedance mismatching between air and absorber when the electromagnetic wave propagates to the foams. Thus, the electromagnetic wave easily radiates into the interior of foams, resulting in a low reflection on the surface. Then, the entered electromagnetic microwaves are reflected among these micropores and their walls in the composite foams, which is converted into heat energy to be dissipated [74]. The conductive linkages of CNT/Ni@CNT/PIL act as an inter-transporting charge carriers for tunneling more electrons and also enhancing the hopping mechanism within the shielded foam material [75]. The abundant terminal groups and the large number of charge carriers of CNT/Ni@CNT/PIL could interact with the incident waves and dissipate them by converting to heat [76]. In addition, the polarization loss, which was caused by interfacial polarization between TPU substrates and conductive fillers, conduction loss caused by conductive network of fillers and magnetic loss caused by Ni@CNTs, synergistically attenuate the microwave energy. Therefore, these results confirm that $\mathrm{SE}_{\mathrm{A}}$ plays a primary role in the $\mathrm{SE}_{\mathrm{T}}$ of composite foams.

Figure 8 shows the specific EMI SE (i.e., EMI SE divided by the sample density and thickness, SSE) of TPU/CNTs/ $\mathrm{Ni@CNTs/PIL} \mathrm{with} \mathrm{various} \mathrm{filler} \mathrm{concentrations} \mathrm{and}$ an evaporate time of $120 \mathrm{~min}$ at $9 \mathrm{GHz}$. The densities of TPU/15CNTs/5Ni@CNTs/PIL, TPU/10CNTs/10Ni@ CNTs/PIL and TPU/5CNTs/15Ni@CNTs/PIL composites are $0.30,0.33$ and $0.35 \mathrm{~g} \mathrm{~cm}^{-3}$, respectively. The density of TPU/10CNTs/10Ni@CNTs/PIL increases with increasing the Ni@CNTs loading. The morphology of composite gradually becomes dense with the increase in the Ni@CNTs content (Fig. S7) because Ni@CNTs are easier to agglomerate than CNTs and the density of Ni@CNTs is greater than that of CNTs. The compressive strength of TPU/CNTs/Ni@ CNTs/PIL is slightly decreased with increasing the Ni@ CNTs content (Figs. S8 and S9). The highest specific EMI SE of TPU/20CNTs/PIL and TPU/10CNTs/10Ni@CNTs/ PIL reaches up to 187.2 and $211.5 \mathrm{~dB} /\left(\mathrm{g} \mathrm{cm}^{-3}\right)$, respectively.

Figure 9 summarizes the EMI SE and SSE of the foamsbased EMI shielding materials reported in the literatures. Table S4 shows the detailed results of the EMI performance.

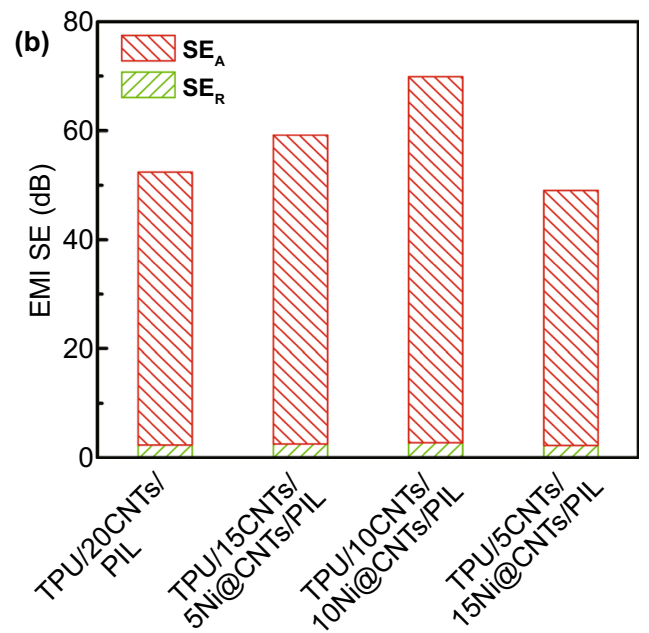

Fig. 7 a EMI SE of TPU/CNTs/Ni@CNTs/PIL foams; $\mathbf{b}$ comparison of $\mathrm{SE}_{\mathrm{T}}, \mathrm{SE}_{\mathrm{A}}$ and $\mathrm{SE}_{\mathrm{R}}$ of TPU/CNTs/Ni@CNTs/PIL foams with 120 min evaporate time and various concentrations at $9.0 \mathrm{GHz}$ 


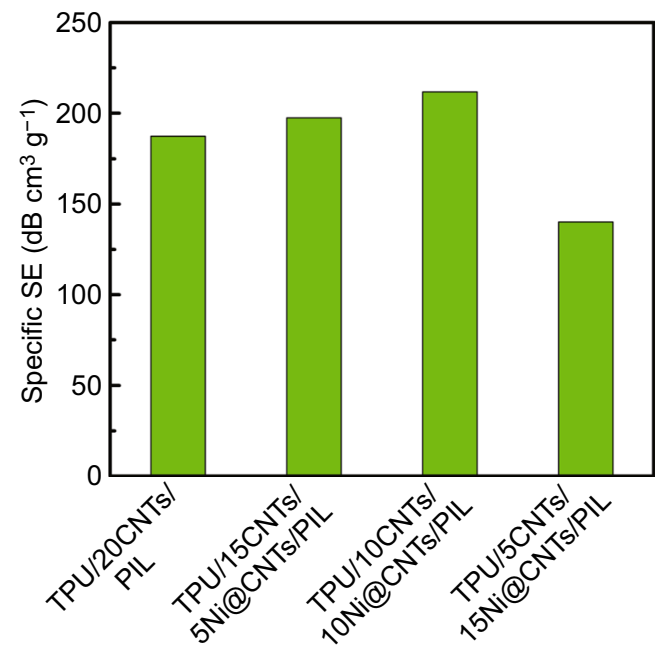

Fig. 8 Specific EMI SE of TPU/CNTs/Ni@CNTs/PIL composite foams with various filler concentrations and the evaporate time of $120 \mathrm{~min}$ at $9.0 \mathrm{GHz}$

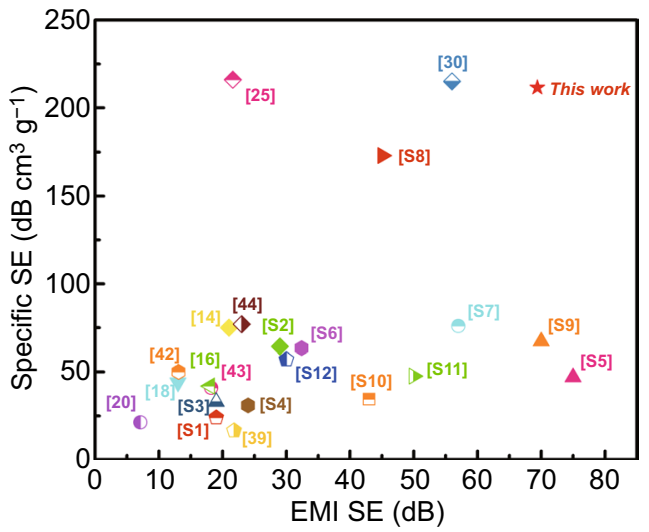

Fig. 9 Comparison of EMI shielding performance of various composite foams ever reported
It is indicated that the TPU/10CNTs/10Ni@CNTs/PIL foams show a very high SSE $\left(211.5 \mathrm{~dB} \mathrm{~cm}^{3} \mathrm{~g}^{-1}\right)$. The high SE and SSE for the foams derive from its particular microcellular structure, which is schematically illustrated in Fig. 10. The TPU/10CNTs/10Ni@CNTs/PIL foam has high electrical conductivity and magnetic permeability [63]. These CNTs possess large specific surface areas and high electrical conductivity. PIL-modified CNTs can wet and interact with PU matrix, which are essential for interfacial polarization and re-reflections. The magnetic loss is due to the effect of magnetic filler Ni@CNT, which introduce more and more magnetic dipoles into the PU matrix. In addition, the uniform dispersion of CNTs and Ni@CNTs increases the interface area between fillers and TPU matrix, which increases the interface polarization and causes the interface polarization dielectric loss [77]. The small-sized microcellular structure adds extensive interfaces and enhances the attenuation of incident electromagnetic waves via multiple reflections [78]. Thus, the composite foams suggest a great potential in applications for aircraft and spacecraft field, which has the characteristics of lightweight and efficient specific EMI SE.

\section{Conclusion}

Lightweight microcellular TPU/CNTs/Ni@CNTs/PIL composite foams with high EMI SE are fabricated with an environmentally benign and efficient NIPS technique. TPU/ CNTs/PIL composite foams have a higher EMI SE value. This is attributed to the PIL coating on the surface of CNTs, which reduces the surface energy and increases the interface areas between the TPU substrates and the fillers. Moreover,

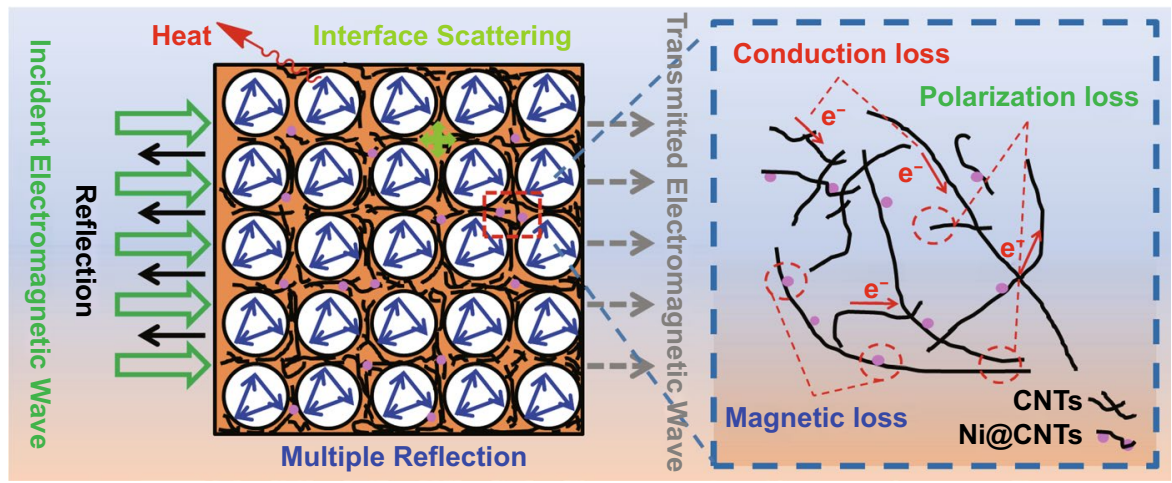

Fig. 10 Schematic description of the microwave transfer across the TPU composite foams 
the evaporate time regulates the microcellular structure and obviously improves electrical conductivity and EMI shielding properties. The EMI SE of TPU/10CNTs/10Ni@CNT/ PIL foam is $69.9 \mathrm{~dB}$ compared with TPU/20CNTs/PIL (53.3 dB) at the same CNTs content. The highest specific EMI SE of TPU/20CNTs/PIL and TPU/10CNTs/10Ni@ CNTs/PIL reaches up to 187.2 and $211.5 \mathrm{~dB} /\left(\mathrm{g} \mathrm{cm}^{-3}\right)$, respectively. The corresponding conduction and magnetic loss, polarization loss in the TPU/10CNTs/10Ni@CNTs/PIL nanocomposites attenuate the electromagnetic waves. The composite foams, which have the characteristics of lightweight and efficient specific EMI SE, show great potential applications in aviation and electronic industrial field.

Acknowledgements This research was supported by the National Natural Science Foundation of China (51603060 and 51673056) and the Industrial Guidance Fund Project of Bengbu City and Hefei University of Technology (JZ2020YDZJ0334).

Open Access This article is licensed under a Creative Commons Attribution 4.0 International License, which permits use, sharing, adaptation, distribution and reproduction in any medium or format, as long as you give appropriate credit to the original author(s) and the source, provide a link to the Creative Commons licence, and indicate if changes were made. The images or other third party material in this article are included in the article's Creative Commons licence, unless indicated otherwise in a credit line to the material. If material is not included in the article's Creative Commons licence and your intended use is not permitted by statutory regulation or exceeds the permitted use, you will need to obtain permission directly from the copyright holder. To view a copy of this licence, visit http://creativecommons.org/licenses/by/4.0/.

Supplementary Information The online version contains supplementary material available at https://doi.org/10.1007/ s40820-021-00677-5.

\section{References}

1. P.T. Xie, Y. Liu, M. Feng, M. Niu, C. Liu et al., Hierarchically porous $\mathrm{Co} / \mathrm{C}$ nanocomposites for ultralight high-performance microwave absorption. Adv. Compos. Hybrid Mater. 4, 173185 (2021). https://doi.org/10.1007/s42114-020-00202-z

2. N. Wu, W. Du, Q. Hu, S. Vupputuri, Q. Jiang, Recent development in fabrication of Co nanostructures and their carbon nanocomposites for electromagnetic wave absorption. Eng. Sci. 13, 11-23 (2021). https://doi.org/10.30919/es8d1149

3. L. Lyu, J. Liu, H. Liu, C. Liu, Y. Lu et al., An overview of electrically conductive polymer nanocomposites toward electromagnetic interference shielding. Eng. Sci. 2, 26-42 (2018). https://doi.org/10.30919/es8d615
4. H.H. Hu, H.P. Liu, D.J. Zhang, J.J. Wang, G.W. Qin et al., $\mathrm{pH}$ and electromagnetic dual-remoted drug delivery based on bimodal superparamagnetic $\mathrm{Fe}_{3} \mathrm{O}_{4} @$ porous silica nanoparticles. Eng. Sci. 2, 43-48 (2018). https://doi.org/10. 30919/es8d136

5. J. Guo, Z. Qu, B. Wang, A reverse thermal cloak design method based on inverse problem theory. ES Energy Environ. 7, 71-83 (2020). https://doi.org/10.30919/esee8c375

6. P. Song, B. Liu, H. Qiu, X.T. Shi, D.P. Cao et al., MXenes for polymer matrix electromagnetic interference shielding composites: A review. Compos. Commun. 24, 100653 (2021). https://doi.org/10.1016/j.coco.2021.100653

7. N. Yousefi, X.Y. Sun, X.Y. Lin, X. Shen, J.J. Jia et al., Highly aligned graphene/polymer nanocomposites with excellent dielectric properties for high performance electromagnetic interference shielding. Adv. Mater. 26, 5480-5487 (2015). https://doi.org/10.1002/adma.201305293

8. Y.H. Zhan, J. Wang, K.Y. Zhang, Y.C. Li, Y.Y. Meng et al., Fabrication of a flexible electromagnetic interference shielding $\mathrm{Fe}_{3} \mathrm{O}_{4} @$ reduced graphene oxide/natural rubber composite with segregated network. Chem. Eng. J. 344, 184-193 (2018). https://doi.org/10.1016/j.cej.2018.03.085

9. W.X. Yang, B.W. Shao, T.Y. Liu, Y.Y. Zhang, R. Huang et al., Robust and mechanically and electrically self-healing hydrogel for efficient electromagnetic interference shielding. ACS Appl. Mater. Interfaces 10(9), 8245-8257 (2018). https://doi.org/10.1021/acsami.7b18700

10. L.C. Jia, D.X. Yan, X.F. Liu, R.J. Ma, H.Y. Wu et al., Highly efficient and reliable transparent electromagnetic interference shielding film. ACS Appl. Mater. Interfaces 10(14), 11941-11949 (2018). https://doi.org/10.1021/acsami.8b004 92

11. B. Zhao, J. Deng, R. Zhang, L. Liang, B. Fan et al., Recent advances on the electromagnetic wave absorption properties of Ni based materials. Eng. Sci. 3, 5-40 (2018). https://doi. org/10.30919/es8d735

12. P. Song, B. Liu, C. Liang, K. Ruan, H. Qiu et al., Lightweight, flexible cellulose-derived carbon aerogel@reduced graphene oxide/PDMS composites with outstanding EMI shielding performances and excellent thermal conductivities. Nano-Micro Lett. 13, 91 (2021). https://doi.org/10.1007/ s40820-021-00624-4

13. Z.P. Chen, C. Xu, C.Q. Ma, W.C. Ren, H.M. Cheng, Lightweight and flexible graphene foam composites for high-performance electromagnetic interference shielding. Adv. Mater. 25, 1296-1300 (2013). https://doi.org/10.1002/adma.201204196

14. Z. Zeng, M. Chen, H. Jin, W. Li, X. Xue et al., Thin and flexible multi-walled carbon nanotube/waterborne polyurethane composites with high-performance electromagnetic interference shielding. Carbon 96, 768-777 (2016). https://doi.org/ 10.1016/j.carbon.2015.10.004

15. R. Asmatulu, P.K. Bollavaram, V.R. Patlolla, I.M. Alarifi, W.S. Khan, Investigating the effects of metallic submicron and nanofilms on fiber-reinforced composites for lightning strike protection and EMI shielding. Adv. Compos. 
Hybrid Mater. 3, 66-83 (2020). https://doi.org/10.1007/ s42114-020-00135-7

16. X. Lv, Y. Tang, Q. Tian, Y. Wang, T. Ding, Ultra-stretchable membrane with high electrical and thermal conductivity via electrospinning and in-situ nanosilver deposition. Compos. Sci. Technol. 200, 108414 (2020). https://doi.org/10.1016/j. compscitech.2020.108414

17. X. Yan, J. Liu, M.A. Khan, S. Sheriff, S. Vupputuri et al., Efficient solvent-free microwave irradiation synthesis of highly conductive polypropylene nanocomposites with lowly loaded carbon nanotubes. ES Mater. Manuf. 9, 21-33 (2020). https:// doi.org/10.30919/esmm5f716

18. J. Chen, Y. Zhu, Z. Guo, A.G. Nasibulin, Recent progress on thermo-electrical properties of conductive polymer composites and their application in temperature sensors. Eng. Sci. 12, 13-22 (2020). https://doi.org/10.30919/es8d1129

19. Y. Zhang, K. Ruan, X. Shi, H. Qiu, Y. Pan et al., $\mathrm{Ti}_{3} \mathrm{C}_{2} \mathrm{~T}_{\mathrm{x}} / \mathrm{rGO}$ porous composite films with superior electromagnetic interference shielding performances. Carbon 175, 271-280 (2021). https://doi.org/10.1016/j.carbon.2020.12.084

20. L. Wang, P. Song, C.T. Lin, J. Kong, J.W. Gu, 3D shapeable, superior electrically conductive cellulose nanofibers $/ \mathrm{Ti}_{3} \mathrm{C}_{2} \mathrm{~T}_{\mathrm{x}}$ MXene aerogels/epoxy nanocomposites for promising EMI shielding. Research 2020, 4093732 (2020). https://doi.org/10. 34133/2020/4093732

21. A. Ameli, P.U. Jung, B.C. Park, Electrical properties and electromagnetic interference shielding effectiveness of polypropylene/carbon fiber composite foams. Carbon 60, 379-391 (2013). https://doi.org/10.1016/j.carbon.2013.04.050

22. Z.H. Zeng, H. Jin, M.J. Chen, W.W. Li, L.C. Zhou et al., Lightweight and anisotropic porous MWCNT/WPU composites for ultrahigh performance electromagnetic interference shielding. Adv. Funct. Mater. 26, 303-310 (2016). https://doi.org/10. 1002/adfm.201503579

23. L. Wang, X.T. Shi, J.L. Zhang, Y.L. Zhang, J.W. Gu, Lightweight and robust $\mathrm{rGO}$ /sugarcane derived hybrid carbon foams with outstanding EMI shielding performance. J. Mater. Sci. Technol. 52, 119-126 (2020). https://doi.org/10.1016/j.jmst. 2020.03.029

24. A. Bianco, S. Burg, A.J. Parnell, C. Fernyhough, A. Washington et al., Control of the porous structure of polystyrene particles obtained by nonsolvent induced phase separation. Langmuir 33(46), 13303-13314 (2017). https://doi.org/10. 1021/acs.langmuir.7b02802

25. Y. Gu, R.M. Dorin, U. Wiesner, Asymmetric organic-inorganic hybrid membrane formation via block copolymer-nanoparticle Co-assembly. Nano Lett. 13(11), 5323-5328 (2013). https:// doi.org/10.1021/nl402829p

26. G.R. Guillen, Y. Pan, M. Li, E. Hoek, Preparation and characterization of membranes formed by nonsolvent induced phase separation: a review. Ind. Eng. Chem. Res. 50(7), 3798-3817 (2011). https://doi.org/10.1021/ie101928r

27. Z. Maghsoud, M. Pakbaz, M.H.N. Famili, S.S. Madaeni, New polyvinyl chloride/thermoplastic polyurethane membranes with potential application in nanofiltration. J. Memb. Sci. 541,
271-280 (2017). https://doi.org/10.1016/j.memsci.2017.07. 001

28. H.L. Yang, Z. Yu, P. Wu, H. Zou, P. Liu, Electromagnetic interference shielding effectiveness of microcellular polyimide/in situ thermally reduced graphene oxide/carbon nanotubes nanocomposites. Appl. Surf. Sci. 434, 318-325 (2018). https:// doi.org/10.1016/j.apsusc.2017.10.191

29. Y. Li, X. Pei, B. Shen, W. Zhai, L. Zhang et al., Polyimide/ graphene composite foam sheets with ultrahigh thermostability for electromagnetic interference shielding. RSC Adv. 5, 24342-24351 (2015). https://doi.org/10.1039/C4RA16421K

30. J. Zhao, G. Luo, J. Wu, H. Xia, Preparation of microporous silicone rubber membrane with tunable pore size via solvent evaporation-induced phase separation. ACS Appl. Mater. Interfaces 5(6), 2040-2046 (2013). https://doi.org/10.1021/ am302929c

31. B. Shen, W. Zhai, M. Tao, J. Ling, W. Zheng, Lightweight, multifunctional polyetherimide/graphene@ $\mathrm{Fe}_{3} \mathrm{O}_{4}$ composite foams for shielding of electromagnetic pollution. ACS Appl. Mater. Interfaces 5(21), 11383-11391 (2013). https://doi.org/ 10.1021/am4036527

32. D.R. Tree, T. Iwama, K.T. Delaney, J. Lee, G.H. Fredrickson, Marangoni flows during nonsolvent induced phase separation. ACS Macro Lett. 7(5), 582-586 (2018). https://doi.org/ 10.1021/acsmacrolett.8b00012

33. J. Ling, W. Zhai, W. Feng, B. Shen, J. Zhang et al., Facile preparation of lightweight microcellular polyetherimide/graphene composite foams for electromagnetic interference shielding. ACS Appl. Mater. Interfaces 5(7), 2677-2684 (2013). https:// doi.org/10.1021/am303289m

34. J. Li, G. Zhang, Z. Ma, X. Fan, X. Fan et al., Morphologies and electromagnetic interference shielding performances of microcellular epoxy/multi-wall carbon nanotube nanocomposite foams. Compos. Sci. Technol. 129, 70-78 (2016). https:// doi.org/10.1016/j.compscitech.2016.04.003

35. Z. Zeng, H. Jin, M. Chen, W. Li, L. Zhou et al., Microstructure design of lightweight, flexible, and high electromagnetic shielding porous multiwalled carbon nanotube/polymer composites. Small 13, 1701388 (2017). https://doi.org/10.1002/ smll.201701388

36. Y. Li, B. Shen, X. Pei, Y. Zhang, D. Yi et al., Ultrathin carbon foams for effective electromagnetic interference shielding. Carbon 100, 375-385 (2016). https://doi.org/10.1016/j.carbon. 2016.01.030

37. F. Moglie, D. Micheli, S. Laurenzi, M. Marchetti, V.M. Primiani, Electromagnetic shielding performance of carbon foams. Carbon 50, 1972-1980 (2012). https://doi.org/10.1016/j.carbon.2011.12.053

38. Q. Song, F. Ye, X. Yin, W. Li, H. Li et al., Carbon nanotubemultilayered graphene edge plane core-shell hybrid foams for ultrahigh-performance electromagnetic-interference shielding. Adv. Mater. 29, 1701583 (2017). https://doi.org/10.1002/ adma.201701583

39. X. Ma, B. Shen, L. Zhang, Y. Liu, W. Zhai et al., Porous superhydrophobic polymer/carbon composites for lightweight and self-cleaning EMI shielding application. 
Compos. Sci. Technol. 158, 86-93 (2018). https://doi.org/ 10.1016/j.compscitech.2018.02.006

40. C.H. Cui, D.X. Yan, H. Pang, L.C. Jia, X. Xu et al., A high heat-resistance bioplastic foam with efficient electromagnetic interference shielding. Chem. Eng. J. 323, 29-36 (2017). https://doi.org/10.1016/j.cej.2017.04.050

41. V.D. Punetha, S. Rana, H.J. Yoo, A. Chaurasia, J.T. McLeskey et al., Functionalization of carbon nanomaterials for advanced polymernanocomposites: a comparison study between CNT and graphene. Prog. Polym. Sci. 67, 1-47 (2017). https://doi.org/10.1016/j.progpolymsci.2016.12.010

42. S.T. Hsiao, C.C.M. Ma, H.W. Tien, W.H. Liao, Y.S. Wang et al., Using a non-covalent modification to prepare a high electromagnetic interference shielding performance graphene nanosheet/water-borne polyurethane composite. Carbon 60, 57-66 (2013). https://doi.org/10.1016/j.carbon. 2013.03.056

43. S. Ganguly, S. Ghosh, P. Das, T.K. Das, S.K. Ghosh et al., Poly(N-vinylpyrrolidone)-stabilized colloidal graphene-reinforced poly(ethylene-co-methyl acrylate) to mitigate electromagnetic radiation pollution. Polym. Bull. 77, 2923-2943 (2020). https://doi.org/10.1007/s00289-019-02892-y

44. D.R. Dreyer, S. Park, C.W. Bielawski, R.S. Ruoff, The chemistry of graphene oxide. Chem. Soc. Rev. 39, 228-240 (2010). https://doi.org/10.1039/B917103G

45. L. Liu, Z. Zheng, C. Gu, X. Wang, The poly(urethane-ionic liquid)/multi-walled carbon nanotubes composites. Compos. Sci. Technol. 70, 1697-1703 (2010)

46. S.M. Shang, W. Zeng, X.M. Tao, High stretchable MWNTs/ polyurethane conductive nanocomposites. J. Mater. Chem. 21, 7274-7280 (2011). https://doi.org/10.1039/C1JM10255A

47. A. Chaudhary, S. Kumari, R. Kumar, S. Teotia, B.P. Singh et al., Lightweight and easily foldable MCMB-MWCNTs composite paper with exceptional electromagnetic interference shielding. ACS Appl. Mater. Interfaces 8(16), 10600-10608 (2016). https://doi.org/10.1021/acsami.5b12334

48. J. Abraham, M.P. Arif, P. Xavier, S. Bose, S.C. George et al., Investigation into dielectric behaviour and electromagnetic interference shielding effectiveness of conducting styrene butadiene rubber composites containing ionic liquid modified MWCNT. Polymer 112, 102-115 (2017). https://doi.org/ 10.1016/j.polymer.2017.01.078

49. T. Welton, Room-temperature ionic liquids solvents for synthesis and satalysis. ChemInform 111, 2071-2084 (2010). https://doi.org/10.1002/chin.199946295

50. S. Biswas, G.P. Kar, S. Bose, Tailor-made distribution of nanoparticles in blend structure toward outstanding electromagnetic interference shielding. ACS Appl. Mater. Interfaces 7(45), 25448-25463 (2015). https://doi.org/10.1021/acsami. $5 \mathrm{~b} 08333$

51. J.M. Thomassin, C. Jérômea, T. Pardoen, C. Bailly, I. Huynen et al., Polymer/carbon based composites as electromagnetic interference (EMI) shielding materials. Mat. Sci. Eng. R 74(7), 211-232 (2013). https://doi.org/10.1016/j.mser.2013.06.001
52. Y. Xu, Y. Yang, D.X. Yan, H. Duan, G. Zhao et al., Flexible and conductive polyurethane composites for electromagnetic shielding and printable circuit. Chem. Eng. J. 360, 1427-1436 (2019). https://doi.org/10.1016/j.cej.2018.10.235

53. A.V. Menon, G. Madras, S. Bose, Shape memory polyurethane nanocomposites with porous architectures for enhanced microwave shielding. Chem. Eng. J. 352, 590-600 (2018). https:// doi.org/10.1016/j.cej.2018.07.048

54. W. Zou, J. Huang, W. Zeng, X. Lu, Effect of ethylene-butylacrylate-glycidyl methacrylate on compatibility properties of poly(butylene terephthalate)/thermoplastic polyurethane blends. ES Energy Environ. 9, 67-73 (2020). https://doi.org/ 10.30919/esee $8 \mathrm{c} 180$

55. C. Liu, Q. Yin, X. Li, L. Hao, W. Zhang et al., A waterborne polyurethane-based leather finishing agent with excellent room temperature self-healing properties and wear-resistance. Adv. Compos. Hybrid Mater. 4, 138-149 (2021). https://doi. org/10.1007/s42114-021-00206-3

56. Z. Zeng, M. Chen, Y. Pei, S.S. Shahabadi, B. Che et al., Ultralight and flexible polyurethane/silver nanowire nanocomposites with unidirectional pores for highly effective electromagnetic shielding. ACS Appl. Mater. Interfaces 9(37), 32211-32219 (2017). https://doi.org/10.1021/acsami.7b07643

57. A.V. Menon, G. Madras, S. Bose, Ultrafast self-healable interfaces in polyurethane nanocomposites designed using dielsalder "Click" as an efficient microwave absorber. ACS Omega 3, 1137-1146 (2018). https://doi.org/10.1021/acsomega.7b018 45

58. Q. Jiang, X. Liao, J. Li, J. Chen, G. Wang et al., Flexible thermoplastic polyurethane/reduced graphene oxide composite foams for electromagnetic interference shielding with high absorption characteristic. Compos. Part A Appl. S 123, 310-319 (2019). https://doi.org/10.1016/j.compositesa.2019. 05.017

59. T. Wu, B. Chen, Facile fabrication of porous conductive thermoplastic polyurethane nanocomposite films via solution casting. Sci. Rep. 7, 17470 (2017). https://doi.org/10.1038/ s41598-017-17647-w

60. X. Ji, D. Chen, J. Shen, S. Guo, Flexible and flame-retarding thermoplastic polyurethane-based electromagnetic interference shielding composites. Chem. Eng. J. 370, 1341-1349 (2019). https://doi.org/10.1016/j.cej.2019.03.293

61. J.M. Kim, Y. Lee, M.G. Jang, C. Han, W.N. Kim, Electrical conductivity and EMI shielding effectiveness of polyurethane foam-conductive filler composites. J. Appl. Polym. Sci. 133, 44373 (2016). https://doi.org/10.1002/app.44373

62. H. Zhang, G. Zhang, Q. Gao, M. Tang, Z. Ma et al., Multifunctional microcellular PVDF/Ni-chains composite foams with enhanced electromagnetic interference shielding and superior thermal insulation performance. Chem. Eng. J. 379, 122304 (2020). https://doi.org/10.1016/j.cej.2019.122304

63. H. Zhang, G. Zhang, J. Li, X. Fan, Z. Jing et al., Lightweight, multifunctional microcellular PMMA/ $\mathrm{Fe}_{3} \mathrm{O}_{4} @ \mathrm{MWCNTs}$ nanocomposite foams with efficient electromagnetic interference shielding. Compos. Part A Appl. S 100, 128-138 (2017). https://doi.org/10.1016/j.compositesa.2017.05.009 
64. I. Okada, S. Shiratori, High-transparency, self-standable gelSLIPS fabricated by a facile nanoscale phase separation. ACS Appl. Mater. Interfaces 6(3), 1502-1508 (2014). https://doi. org/10.1021/am404077h

65. R. Rarima, G. Unnikrishnan, Poly(lactic acid)/gelatin foams by non-solvent induced phase separation for biomedical applications. Polym. Degrad. Stabil. 177, 109187 (2020). https://doi. org/10.1016/j.polymdegradstab.2020.109187

66. Y. Li, B. Shen, D. Yi, L. Zhang, W. Zhai et al., The influence of gradient and sandwich configurations on the electromagnetic interference shielding performance of multilayered thermoplastic polyurethane/graphene composite foams. Compos. Sci. Technol. 138, 209-216 (2017). https://doi.org/10.1016/j. compscitech.2016.12.002

67. S. Zhang, J. Zhang, Y. Zhang, Y. Deng, Nanoconfined ionic liquids. Chem. Rev. 117(10), 6755-6833 (2017). https://doi. org/10.1021/acs.chemrev.6b00509

68. Y.J. Wan, P.L. Zhu, S.H. Yu, R. Sun, C.P. Wong et al., Anticorrosive, ultralight, and flexible carbon-wrapped metallic nanowire hybrid sponges for highly efficient electromagnetic interference shielding. Small 14, 1800534 (2018). https://doi. org/10.1002/smll.201800534

69. Z. Ma, S. Kang, J. Ma, L. Shao, Y. Zhang et al., Ultraflexible and mechanically strong double-layered aramid nanofiber$\mathrm{Ti}_{3} \mathrm{C}_{2} \mathrm{~T}_{\mathrm{x}}$ MXene/silver nanowire nanocomposite papers for high-performance electromagnetic interference shielding. ACS Nano 14(7), 8368-8382 (2020). https://doi.org/10.1021/ acsnano.0c02401

70. X. Zhi, J. Liu, H.B. Zhang, S. Hong, Z.Z. Yu, Simultaneous enhancements in electrical conductivity and toughness of selectively foamed polycarbonate/polystyrene/carbon nanotube microcellular foams. Compos. Part B 143, 161-167 (2018). https://doi.org/10.1021/acs.iecr.7b00446

71. G. Sang, P. Xu, C. Liu, P. Wang, X. Hu et al., Synergetic effect of Ni@ MWCNTs and hybrid MWCNTs on electromagnetic interference shielding performances of polyurethane-matrix composite foams. Ind. Eng. Chem. Res. 59(34), 15233-15241 (2020). https://doi.org/10.1021/acs.iecr.0c01766
72. T. Kuang, L. Chang, F. Chen, Y. Sheng, D. Fu et al., Facile preparation of lightweight high-strength biodegradable polymer/multi-walled carbon nanotubes nanocomposite foams for electromagnetic interference shielding. Carbon 105, 305-313 (2016). https://doi.org/10.1016/j.carbon.2016.04.052

73. X.H. Li, X. Li, K.N. Liao, P. Min, T. Liu et al., Thermally annealed anisotropic graphene aerogels and their electrically conductive epoxy composites with excellent electromagnetic interference shielding efficiencies. ACS Appl. Mater. Interfaces 8(48), 33230-33239 (2016). https://doi.org/10.1021/ acsami.6b12295

74. B. Shen, Y. Li, W. Zhai, W. Zheng, Compressible graphenecoated polymer foams with ultralow density for adjustable electromagnetic interference (EMI) shielding. ACS Appl. Mater. Interfaces 8(12), 8050-8057 (2016). https://doi.org/ 10.1021/acsami.5b11715

75. S. Ganguly, P. Das, P.P. Maity, S. Mondal, S. Ghosh et al., Green reduced graphene oxide toughened semi-IPN monolith hydrogel as dual responsive drug release system: rheological, physicomechanical, and electrical evaluations. J. Phys. Chem. B 122, 7201-7218 (2018). https://doi.org/10.1021/acs.jpcb. $8 b 02919$

76. J.Q. Luo, S. Zhao, H.B. Zhang, Z.M. Deng, L. Li et al., Flexible, stretchable and electrically conductive MXene/natural rubber nanocomposite films for efficient electromagnetic interference shielding. Compos. Sci. Technol. 182, 107754 (2019). https://doi.org/10.1016/j.compscitech.2019.107754

77. S. Ghosh, S. Ganguly, S. Remanan, S. Mondal, S. Jana et al., Ultra-light weight, water durable and flexible highly electrical conductive polyurethane foam for superior electromagnetic interference shielding materials. J. Mater. Sci. Mater. El. 29, 10177-10189 (2018). https://doi.org/10.1007/ s10854-018-9068-2

78. C. Wang, J. Li, S. Guo, High-performance electromagnetic wave absorption by designing the multilayer graphene/thermoplastic polyurethane porous composites with gradient foam ratio structure. Compos. Part A Appl. S 125, 105522 (2019). https://doi.org/10.1016/j.compositesa.2019.105522 